\title{
Atom Probe Tomography Analysis of a Gallium-Nitride-Based Commercial Light- Emitting Diode
}

\author{
T.J. Prosa ${ }^{1}$, D. Olson ${ }^{1}$, A.D. Giddings ${ }^{1}$, W Lefebvre $^{2}$, P.H. Clifton ${ }^{1}$ and D.J. Larson ${ }^{1}$ \\ 1. CAMECA Instruments, Inc., 5500 Nobel Drive, Madison, WI USA 53711 \\ 2. Université de Rouen, Saint Etienne du Rouvray 76801 FRANCE
}

Thin-film light-emitting diodes (LEDs) composed of $\mathrm{GaN} / \mathrm{In}_{\mathrm{x}} \mathrm{Ga} \mathrm{1}_{1-\mathrm{x}} \mathrm{N} / \mathrm{GaN}$ quantum well structures (QW) are integrated into modern optoelectronic devices because of a tunable InGaN bandgap which enables emission of the full visible spectrum [1,2]. Key issues related to ultra-efficient emission from these materials include compositional inhomogeneity (defects) and interfacial abruptness. Distribution of indium within the QWs and confinement of indium to the QW region are central to understanding carrier localization and recombination properties affecting device performance; however, the measurement of these structural properties is an ongoing challenge.

Atom probe tomography (APT) offers unique capabilities for 3D nano-electronic device characterization including 3D compositional mapping of a relevant volume $\left(>10^{6} \mathrm{~nm}^{3}\right)$, high detection efficiency $(>50 \%)$, and good sensitivity $[3,4]$. In this study, APT is used to understand the distribution of dopants as well as aluminum and indium alloying agents in a commercial gallium nitride device. Measurements using transmission electron microscopy (TEM) and secondary ion mass spectrometry (SIMS) have also been made to improve the accuracy of the APT analysis as well as contrast the information content of these complimentary techniques.

An OSRAM $455 \mathrm{~nm}$ Golden Dragon ${ }^{\circledR}$ Plus LED was purchased from a retail supplier, depackaged, and fabricated into APT compatible specimens using standard focused-ion-beam methods [5,6]. APT analysis revealed various structures which are captured in the atom map and composition profile shown in Fig. 1. The main features include (from top to bottom in the atom map): Mg doping (to form p-type $\mathrm{GaN}$ ) near the top of the structure, Al-rich electron blocking layer, In-rich multi-QW region, and an Inbased superlattice structure near the bottom of the dataset. The multi-QW region is highlighted in the atom map and composition profile shown in Fig. 2. Here the APT QW composition is compared to the layer thickness and spacing extracted from a high resolution TEM image intensity analysis. The two methods show good agreement. Additional analyses of the various regions within the data will be presented as well, illustrating the feasibility of using APT for both competitive analysis and failure analysis within the commercial LED market.

[1] J. Wu et al., Appl. Phys. Lett. 80 (2002) p.3967.

[2] W. Walukiewicz et al., J. Phys. D-Appl. Phys. 39 (2006) p. R83.

[3] D. J. Larson et al., "Atom Probe Tomography for Microelectronics"; in Handbook of

Instrumentation and Techniques for Semiconductor Nanostructure Characterization, eds. R. Haight, F.

Ross and J. Hannon, (World Scientific Publishing/Imperial College Press) (2011) p. 407.

[4] T. F. Kelly and D. J. Larson, Annual Reviews of Materials Research 42 (2012) p. 1.

[5] D. J. Larson et al., Ultramicroscopy 79 (1999) p. 287.

[6] M. K. Miller et al., Microsc. Microanal. 13 (2007) p. 428. 

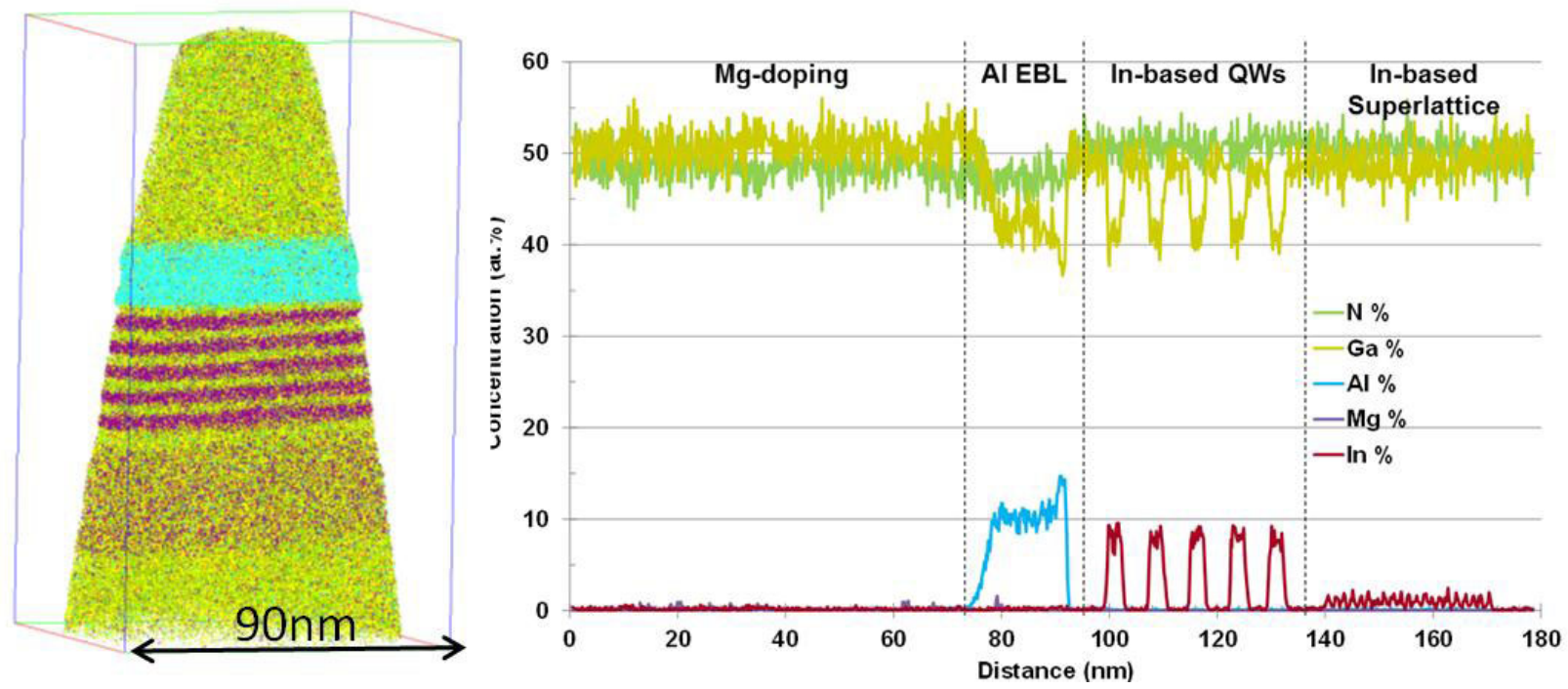

Figure 1. Atom map (left) and concentration profile (right) through a selected region of a GaN-based LED device. The primary structural regions are described at the top of the concentration profile.

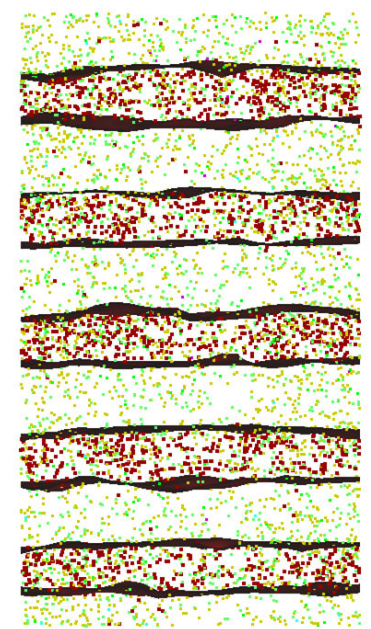

Ga N In

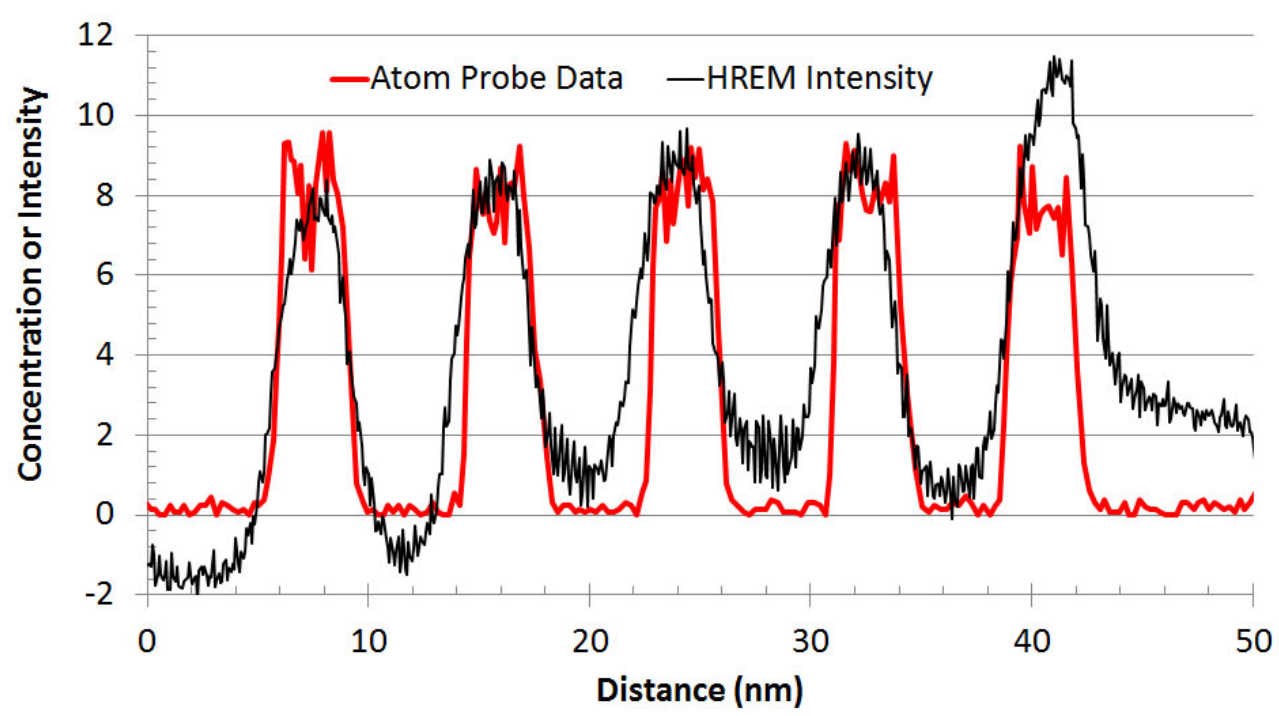

Figure 2. Atom map and iso-indium-concentration surfaces (4 at.\%) (left) are shown enabling measurement of QW thickness via concentration profile (right). Comparison is made with HREM measurements of image intensity (arbitrary units) overlaid on the concentration profile (right). 